\begin{tabular}{ccc}
\hline & International Journal of Engineering \& Technology, $10(2)(2021) 89-94$ \\
SPC & Website: $w w w$. sciencepubco.com/index.php/IJET \\
Research paper & Technology \\
\hline
\end{tabular}

\title{
Compression of medical images by quaternionic wavelet transform
}

\author{
Bertrand Ledoux Ebassa Eloundou ${ }^{1}$ *, Aimé Joseph Oyobe \\ Okassa $^{2}$, Hervé Ndongo Abena ${ }^{3}$, Pierre Ele ${ }^{4}$ \\ ${ }^{1}$ Technologies and Applied Sciences Laboratory (LTSA), University of Douala, Cameroon \\ ${ }^{2}$ Masuku Polytechnic School, Gabon \\ ${ }^{3}$ Energy, Signal, Imaging and Automatic Laboratory (LESIA), University of Ngaoundere, Cameroon \\ ${ }^{4}$ National Higher Polytechnic School of Yaounde (ENSPY), University of Yaounde, Cameroon \\ *Corresponding author E-mail: berteloundou@yahoo.fr
}

\begin{abstract}
Technological developments for several years have resulted in the handling (storing, exchanging or processing) of increasingly important data in various fields and particularly in medical field. In this works we present a new image compression / decompression algorithm based on the quaternion wavelet transform (QWT). This algorithm is simple, fast and efficient. It has been applied to medical images. The results obtained after decompression are appreciated through the compression parameter values of CR, PSNR, and MSE and by visual observation. By the values of these parameters, the results of the algorithm are considered encouraging.
\end{abstract}

Keywords: Quaternionic Wavelet; Compression; Medical Image; PSNR; Compression Ratio.

\section{Introduction}

Image compression can be defined as reducing the size of an image file, while maintaining an acceptable level of quality. It proceeds by reducing data redundancy of an image. The objective is to reduce the original size of the image. This compression allows the image to be stored without taking up a lot of space or transmitting it faster. Several works have been carried out to meet the compression needs. This work is mainly aimed at increasing compression rates, improving the quality of the reconstructed signal and reducing the complexity of existing compression algorithms [1]. This remarkable efficiency is essentially due to two properties which are the parsimony of representation and the whitening of data [2]. Several compression algorithms have been developed, each effective for a specific type of data and particular for specific images. We propose in this work a new image compression algorithm using wavelet transform quaternion. The use quaternions for modeling digital images has allowed the development of analysis and processing tools specifically dedicated to these vector images (used for images in which each pixel is a vector). [3] This work does not directly aim a comparative study with the other transforms, but the compression of medical images by the quaternion wavelet transform. So for our work, we present the general compression of the properties of quaternions, the proposed method and tests on medical images, and finally the presentation and analysis of results.

\section{State of the art on image compression}

\subsection{Compression generalities}

The digital representation of images gives problems of storage and / or transmission. A solution to these problems partly resides in the development and improvement of compression algorithms. This becomes necessary given the large volume of information implemented when using images [4 - 6]. According to the quality of the reconstructed image, there are lossless and lossy compressions of data. In a lossless compression algorithm, the reconstructed signal is identical to the original signal. This type of compression does not give high compression rates and therefore cannot be used in complex cases such as image compression. The most used methods in this case, are coding repetitions, entropy coding algorithms and adaptive dictionary such as LZW more suitable text-based information, scanners and fax machines [4 - 7]. Lossy compression techniques are used when a number of non-essential details can be removed in the signal. This type of compression gives high compression ratios. The most used methods are the one called by transform: the discrete cosines transform, discrete wavelets, fractals, Walsh-Hadamard and the Karhunen-Loeve [1], [8 - 10]. Many images compression algorithms in general and in the medical field in particular have been implemented, which have made it possible to reduce the size of the data stored and / or to optimize the bandwidth, while retaining important diagnostic data. Ragab and al. showed that analytical transformations have an important role in decorrelation of grayscale images, energy compaction of the image and hardly affect the performance of the compression technique. They discussed the importance of image compression coding systems by comparing the bit rate reduction capability and signal-to-noise 
ratio of five transforms, namely the Karhunen-Loeve transform (KLT) to the cosine transform discrete transform (DCT), Hartly transform (DHT), discrete Gabor transform (DGT) and discrete wavelet transform (DWT) [11]. Comparative exploitation of compression methods such as Discrete Cosine Transform (DCT), Wavelet Transform (DWT), wavelet packets and b-splines shows that the b-spline compression technique is more suitable for ElectroMyographic (EMG) signal compression [12]. Sanjay J. Bagul and al. introduced a hybrid image compression algorithm combining lossy discrete cosine transformation (DCT) and lossless Run Length Encoding (RLE), Huffman and Lempel-Ziv-Welch (LZW) techniques, thus achieving better compression [7]. C. Mandhu and al. have proposed a compression algorithm based on quaternion wavelets, which results in a substantial reduction in compression ratio (CR) and better medical image execution time without a huge lack of information compared to the Complex Wavelet Transform (CWT) and the Discrete Wavelet Transform (DWT), with relatively low compression rates [13]. Raphaël Soulard and al. used the Quaternionic Wavelet Transform (QWT) to separate the information contained in an image more efficiently than a classical wavelet transform, they used the QWT to achieve better classification performance than with the DWT [14]. Boukli Hacene Ismail proposed a new transformation called quinconce wavelets (QcWT second generation wavelet), more efficient than the classical wavelet (DWT) which he applied with the SPIHT encoder on these same images and obtains very satisfactory and encouraging results compared to many of the best coders cited in the literature. Although he obtains promising visual results which are confirmed by evaluation parameters such as PSNR, MSSIM, TFC and CR, he believes in prospects that it would be possible to obtain much better results with quaternions or even monogenic wavelets [15]. Very few algorithms use quaternions, the originality of our article is the compression of medical images from a single component (real component) by quaternion transforms.

\subsection{Quaternionic wavelet transformation}

Just as a real signal is conventionally associated with a complex signal, it is possible to immerse a complex signal in a larger space, which is the body of quaternions $\mathrm{H}$. This is a canonical basis of 4-dimensional body $\{1, \mathrm{i}, \mathrm{j}, \mathrm{k}\}$, where $\mathrm{i}, \mathrm{j}, \mathrm{k}$ are pure imaginaries.

$$
i^{2}=j^{2}=k^{2}=-1
$$

With these relationship:

$$
\left\{\begin{array}{c}
i j=-j i=k \\
j k=-k j=i \\
k i=-i k=j \\
i j k=-1
\end{array}\right.
$$

The product is non-commutative, i.e. for $\mathrm{p}, \mathrm{q} \in \mathrm{H}, \mathrm{pq} \neq \mathrm{qp}$ in general. A quaternion can be seen as a quadruplet of real values ( $a, b, c, d)$ following very specific multiplication laws, any quaternion $\mathrm{q}$ is written in Cartesian form as:

$$
q=a+b i+c j+d k
$$

Where $a, b, c, d \in I R$. Its module is:

$|q|^{2}=a^{2}+b^{2}+c^{2}+d^{2}$

But we can also see them as complex numbers with complex coefficients.

$q=z_{1}+z_{2} j$

Where $z_{1}=q_{0}+\mathrm{i} q_{1}$ and $z_{2}=q_{2}+\mathrm{i} q_{3}$ are complex numbers. This quaternion writing form is known as the "Cayley-Dickson notation" [16].

The quaternionic wavelet transformation (QWT) provides a multiscale analysis whose coefficients are 2D analytical, with an amplitude almost invariant by translation and a phase composed of three angles, moreover the phase (diagonal component) has a clear advantage because its measurements supplement the information with new characteristics of "quantity of diagonal structures", which allows it to outperform the discrete wavelet transform (DWT) and complex wavelets. The QWT integrates the notion of phase in a wavelet decomposition. Defined from an analytical quaternionic mother wavelet, the QWT outputs quaternionic coefficients whose phase precisely describes the encoded structures. The power of image description already provided by the decomposition sub-band is then supplemented by a more detailed description through the phase. Each sub-band of the QWT can be seen as the analytical signal associated with a narrowband of the image. The amplitude of a coefficient QWT |q |, invariant by image translation, quantifies the presence of a component at any spatial position in each frequency sub-band. The quaternion scale function and the corresponding real function additives of the wavelet QWT are given by the following matrix:

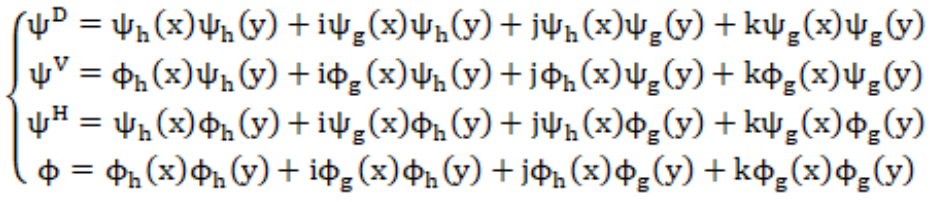

Where $\psi^{\mathrm{D}}, \psi^{\mathrm{V}}, \psi^{\mathrm{H}}$ represent the wavelet functions and $\phi$ represent the scale functions.

This allows us to obtain the quaternionic decomposition (Analysis) and reconstruction (Synthesis) structure of an image $\mathrm{X}$ given by Figure 1 below: 
(A)

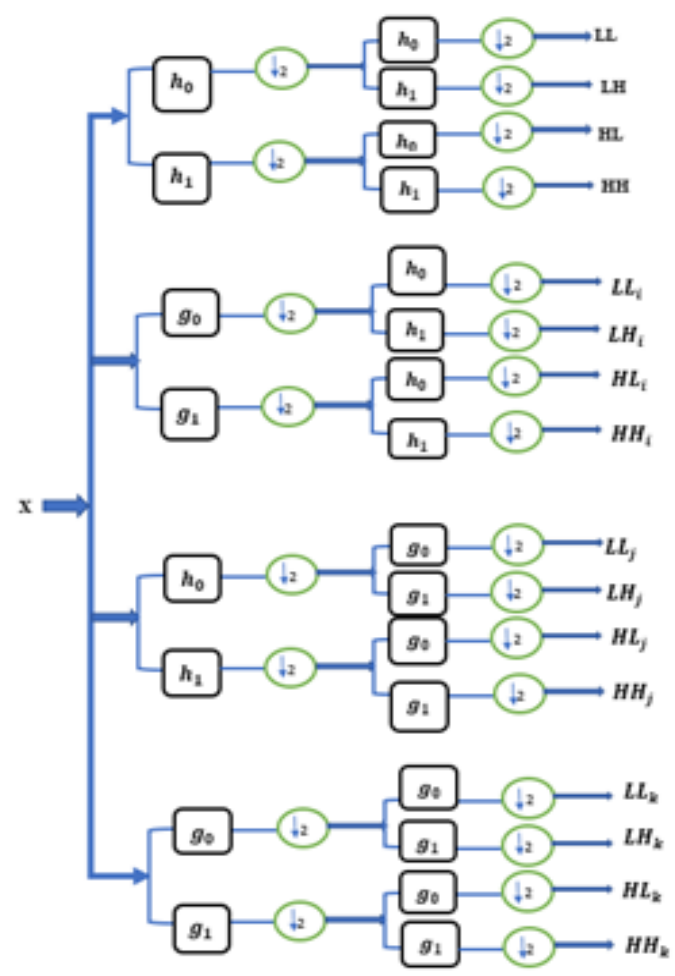

(B)

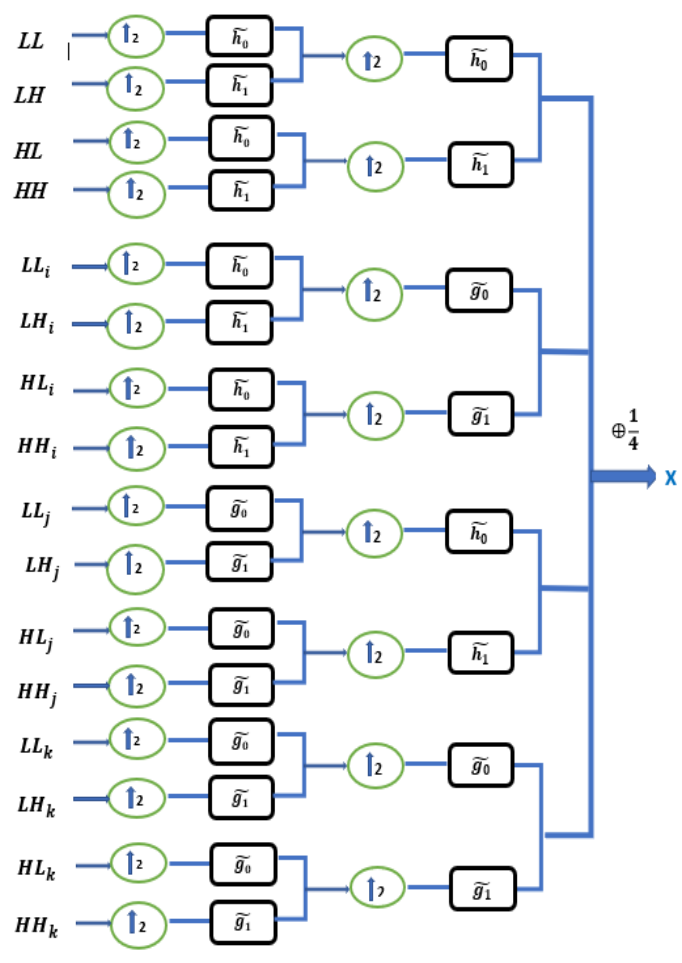

Fig. 1: Decomposition and Reconstruction Structure of Quaternion Filter Banks [13].

Two complementary filter banks, one using an even filter, the other an odd filter, lead to four separable 2D filter banks, slightly offset from each other. These shifts correspond theoretically to the phase shifts of the 2D Hilbert transforms. One obtains a sub-pixel precision, translated indirectly in the notion of phase [17]. The original image $\mathrm{X}$ is decomposed as shown in (a) by a set of quaternion filter banks connected by operators $\downarrow 2$ of data subsampling (downsampling), low-pass $\left(h_{i}\right)$ and high-pass $\left(g_{i}\right)$ filters for the analysis (i taking the values 0 and 1) the signs of interpolation. These quaternion filters allow us to obtain the 4 components of quaternions: a real part and three imaginary parts. Each component consists of 4 sub-bands LL, LH, HL and HH. Likewise, in order to reconstruct the filter banks and the decomposed image, we use the reconstruction structure (Synthesis) given by (b), a phase of dilation of the data with insertion of zeros (upsampling), obtained using of the operator $\uparrow 2$ precedes the low-pass $\left(\widetilde{h_{2}}\right)$ and high-pass $\left(\widetilde{g}_{2}\right)$ filtering operations (i taking the values 0 and 1$)$ allows us to obtain from our four components (real and 3 imaginary) the reconstructed image.

\subsection{Evaluation criteria}

Two groups of criteria are used to evaluate a compression algorithm, namely quantitative criteria and qualitative criteria. In our work, we will focus on:

- Compression ratio: The compression ratio is a quantitative measure of the performance of a data compression algorithm. Usually expressed as a percentage, and defined by:

$C R=\left(1-\frac{\text { Number of bits of the compressed image }}{\text { Number of bits of the original image }}\right) * 100$

- Distortion measurement: Distortion (D) is the error introduced by the compression operation, due to the fact that possibly the reconstructed image is not exactly identical to the original image. The measure of distortion generally used in image compression is the root mean square error MSE so the expression is given as follows:

$\operatorname{MSE}^{2}=\frac{1}{M * N} \sum_{i=1}^{N} \sum_{j=1}^{M}(I(i, j)-\hat{I}(i, j))^{2}$

- The peak signal to noise ratio (PSNR) given by:

PSNR $=10 \log _{10}\left(\frac{\text { dynamique de limage }}{\text { MSE }}\right)^{2}$

Where I (i, j) represents the original image of size $\mathrm{MxN}$, and $\hat{I}(\mathrm{i}, \mathrm{j})$, the reconstructed image.

\section{Proposed compression method}

\subsection{Materials and tools}


The algorithms were implemented using MATLAB 2018a software installed on a microcomputer with 4GB of RAM, 500G of hard disk and $3 * 2.5 \mathrm{GHz}$ frequency with a Windows 10 Professional edition environment. The images used in this work are grayscale and color medical images (converted to grayscale), of dimension $128 * 128,256 * 256$ and $512 * 512$ pixels, of different complexities and structures. Obtained from: medicalxpress.com, Paris Imaging Center and the Cyst Science Photo Library.

(A)

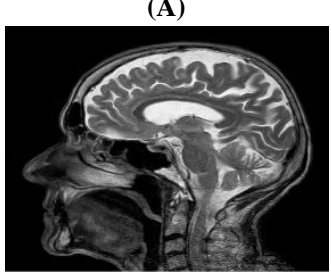

(E)

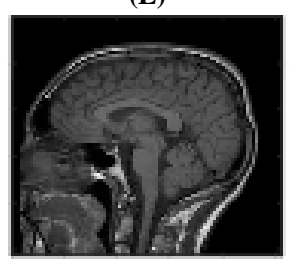

(B)

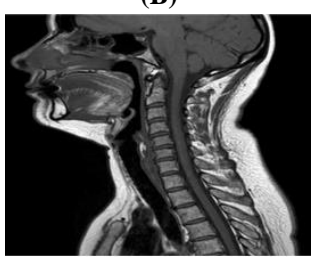

(F)

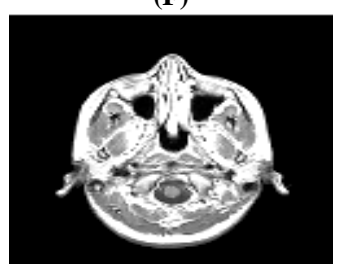

(C)

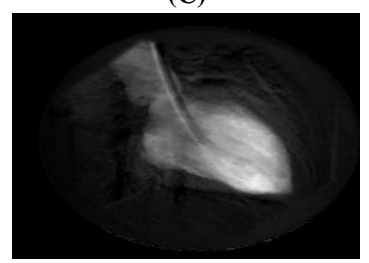

(G)

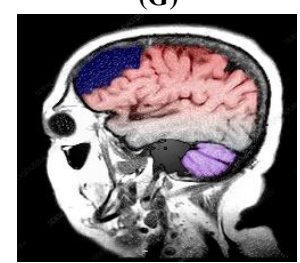

(D)

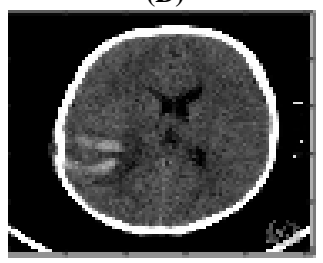

Fig. 3: Medical Images Used.

The proposed diagram block of the image compression / decompression process using the quaternion wavelet transform is shown at the figure 4 .

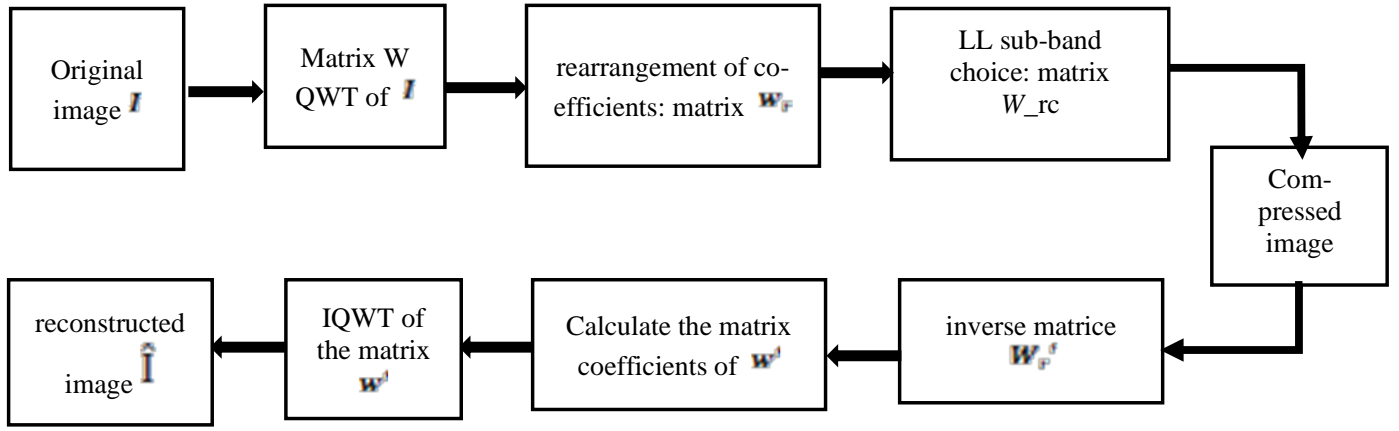

Fig. 4: Proposed Compression / Decompression Scheme.

\subsection{Image transformation process}

Consider the original image I, which is a medical image (MRI, Scanner, etc.) of size $\mathrm{m} * \mathrm{n}$ pixels. The image transformation and reconstruction block diagram is shown in Figure 4, and is summarized by the following steps:

1) Let us decompose the original image I by the quaternionic wavelet transform in $\mathrm{J}$ resolution levels. We thus obtain (3J + 1) quaternionic sub-bands (LL, LH, HL, HH) and $(3 \mathrm{~J}+1) * 4$ elements constituting the matrix w of the QWT due to the fact that each subband is composed of 4 elements.

2) we calculate each sub-band module from the QWT elements in order to obtain the standard format representing w_r matrix of wavelet transforms;

$w_{r}=\left(\begin{array}{ll}L L & L H \\ H L & H H\end{array}\right)$

3) Eliminate the three sub-bands LH, HL, HH and keep only the sub-band LL among the details of sub-bands of the last resolution level J, consisting of: (LLR LL $\varphi$, LL $\theta$, LL $\psi$ );

4) Calculate the new w_rc matrix and display the corresponding images which represent the matrix of the selected sub-band and the compressed image;

$w_{-r e}=\left(\begin{array}{ll}L L_{r} & L L_{\varphi} \\ L L_{\theta} & L L_{\Psi}\end{array}\right)$

\subsection{The image reconstruction process}

1) Determine the coefficients of the inverse matrix W_r 'of the selected sub-band W_rc.

$w_{r}{ }^{\prime}=\left(\begin{array}{ll}L L^{\prime} & L H^{\prime} \\ H L^{\prime} & H H^{\prime}\end{array}\right)$

With $L L^{\prime}=$ inverse $L L_{r}, L H^{\prime}=$ inverse $L L_{\varphi}, H L^{\prime}=$ inverse $L L_{\theta}$ et $H H^{\prime}=$ inverse $L L_{\Psi}$.

2) Calculate the elements of the new matrix w' from the matrix w_r' made up of the selected and modified sub-band w_rc. 
3) Determine the reconstructed image $\hat{I}$ by calculating the inverse of quaternion wavelet transform (IQWT) w'.

\section{Results and discussions}

Applying our algorithm to medical images allowed us to obtain results evaluated on the basis of parameters such as compression rate, root mean square error (MSE) and peak signal-to-noise ratio (PSNR).

a) IRM of centre paris $19512 * 512 \mathrm{jpg}$
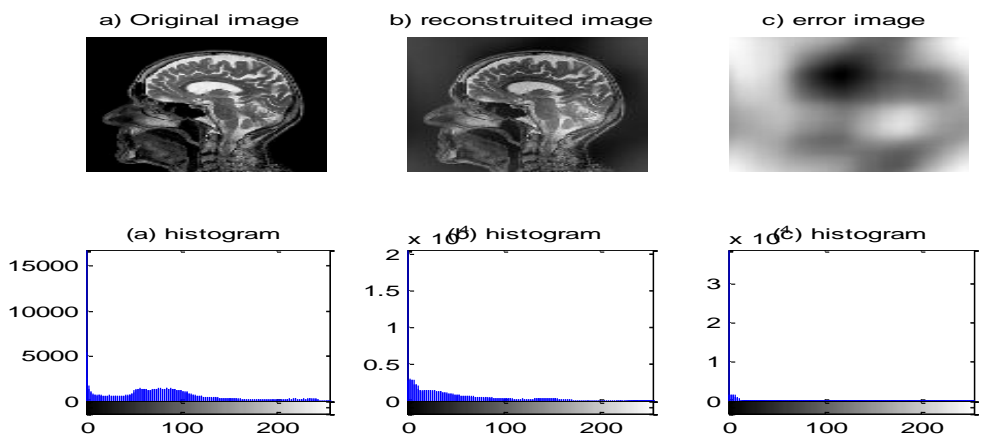

b) Cervical IRM jpg 512*512
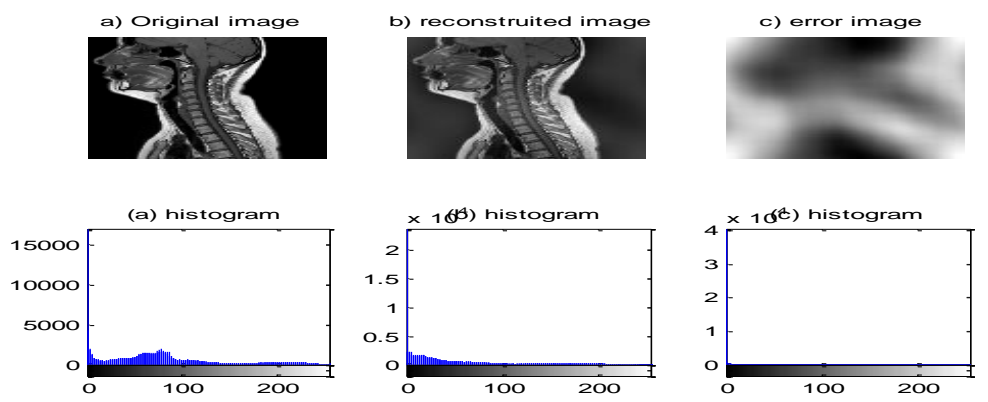

c) Heart_001 tiff $256 * 256$
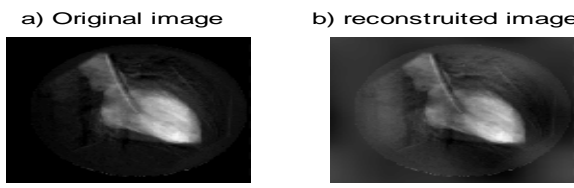

c) error image

(a) histogram

(b) histogram
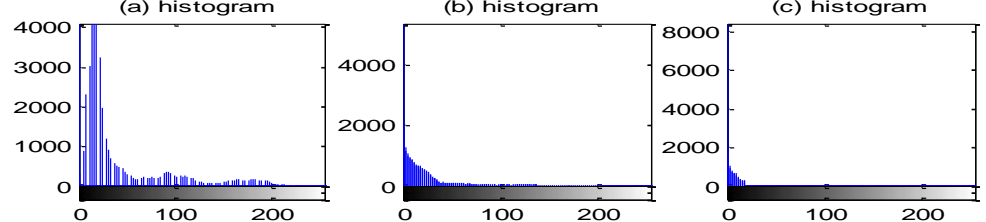

Fig. 5: Original Images, Reconstructed Images, Error Image and Respective Histograms.

The results of the compression / decompression of the images in Figure 5 are reported in the table 1.

Table 1: Evaluation of Image Compression Parameters

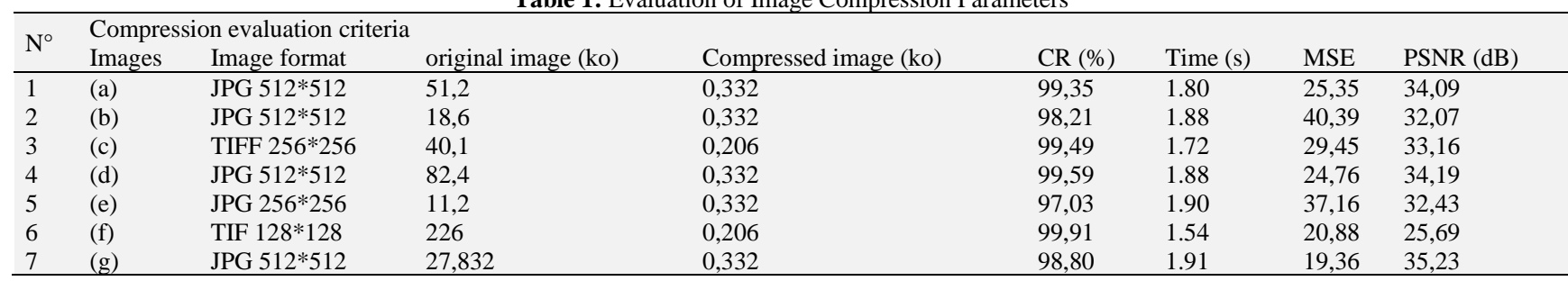

Table 1 shows the different values obtained, the sizes of the original images, the compression ratio, running time of the algorithm, the mean square error and peak signal to noise ratio. Implementation of our algorithm allowed us to obtain very good compression rates. These compression ratios vary between $97.05 \%$ and $99.90 \%$. The MSE is very low, which justifies the good quality of the image reconstruction. In terms of energy, PSNR shows that this algorithm allows good conservation of the energy of the signal (image). Execution times of the order of 1 to 2 seconds are an indication of the ease of execution of the algorithm and its low computational load. These good results are obtained through the multiscale decomposition of wavelets in general and quaternionic wavelets in particular. 


\section{Conclusion}

This work presents a new stable, simple and robust algorithm for compressing / decompressing grayscale medical images, based on the quaternion wavelet transform with a single component. This modified quaternion wavelet decomposition allowed us to obtain a reconstructed image from the only real component. The QWT has thus helped remove maximum redundancy due to the other sub-bands (LH, $\mathrm{HH}, \mathrm{HL}$ ). Our algorithm allows not only to obtain very good results in terms of compression ratio, PSNR and MSE, but also simplicity of implementation and relatively short execution time. Moreover, our algorithm can better preserve fine structures and reproduces images reconstructed good qualities almost identical to the original image, which is of great importance for applications in medicine in particular and in many others areas in general. In the following, our algorithm can be tested on others signals.

\section{References}

[1] Aime Joseph Oyobe Okassa, Joseph Mvogo Ngono, Pierre Ele, «compresion of the EMG signals by Walsh-Hadamard transform associated with the predictive coding DPCM», International Journal of System Signals Control and Engineering Application 12(1): Pg 1-7, 2019. ISSN: 1997-5422. https://doi.org/10.36478/ijssceapp.2019.1.7

[2] Brahmi Sarah et benziane Zahra, Université Abou Bakr Belkaïd de Tlemcen, «La compression des images médicales sous un Smartphone Android », Faculté de Technologie, Département de Génie Biomédical ; mémoire de projet de fin d'études Soutenu le 20 Septembre 2017.

[3] Nicolas Le Bihan, « Traitement quaternionique des images couleur », LIS - UMR CNRS 5083961 Rue de la Houille Blanche Domaine Universitaire, BP 4638402 Saint Marin d'H’eres Cedex. Nicolas.Le-Bihan@lis.inpg.fr.

[4] Aimé Joseph Oyobe-Okassa; Dieudonné Abessolo Assoumou; Pierre Ele, «Compression of EMG signals by superimposing Methods: Case of WPT and TCD», International Journal of Engineering and Technology (IJET); p1335-1343; vol 8 N² Apr-May 2016.

[5] Pratishtha Gupta, G.N Purohit, Varsha Bansal, « A Survey on Image Compression Techniques», International Journal of Advanced Research in Computer and Communication Engineering Vol. 3, Issue 8, August 2014 ISSN (Online) : 2278-1021 ISSN (Print) : $2319-5940$.

[6] Hadjem Karima Et Ziad Malha, « Etude comparative de méthodes de compression d'images basées sur les codeurs par plans de bits », Faculté du Génie Electrique et D'informatique Département d'Electronique, Mémoire de fin d'études de Master soutenu publiquement le 28/09/2016.

[7] Sanjay J. Bagul , Navinchandra G.Shimpi, Pradeep M.Patil, « JPEG Image Compression Using Fast 2-D DCT Technique», International Journal of Advanced Research in Computer and Communication Engineering Vol. 3, Issue 11, November 2014, ISSN (Online) : $2278-1021$ ISSN (Print) : 2319-5940. https://doi.org/10.17148/IJARCCE.2014.31114.

[8] Torres-Urgell, Lynn Kirlin, «Adaptive image compression using Karhunen-Loeve transform», Signal Processing Volume 21, Issue 4, December 1990, Pg 303.313; https://doi.org/10.1016/0165-1684(90)90100-D.

[9] Wai Lam Chan, Hyeokho Choi, Richard Baraniuk, « quaternion wavelets for image analysis and processing», 2004 International Conference on Image Processing, ICIP 2004 -, Singapour.

[10] Djamel Chikouche, Ridha Benzid And Khaled Rouabah, «Application de la DCT et le Codage de Huffman pour la Compression des Images Médicales», SETIT, 5th International Conference: Sciences of Electronic, Technologies of Information and Telecommunications March 2009; Pg 22.26 TUNISIA.

[11] A.S. Ragab, A.S.A. Mohamed, and M.S. Hamid: «Efficiency of analytical transforms for image compression», in Proceedings of the Fifteenth National Radio Science Conference (NRSC), Cairo, 1998, pp. B16/1 - B16/10.

[12] Pascal Eloundou Ntsama, Piere Ele, Emmanuel Tonye, «Compression robuste du signal ElectroMyoGraphique (EMG) par la transformée avec les B-splines », Cari 2004; 7/10/04; 14:50; Pg 59.

[13] C. Madhu, E. Anant Shankar, «Image compression using Quaternion wavelet transform », Helix vol. 8(1): 2691-2695, published on 31st December 2017, Copyright (C) 2018 Helix ISSN 2319 - 5592 (Online). https://doi.org/10.29042/2018-2691-2695.

[14] Boukli Hacene Ismail: «Compression d'images médicales par ondelettes de seconde génération », Thèse Pour obtenir le titre de Docteur En Electronique biomédicale, Université Abou Bekr Belkaid-TLEMCEN-, Département de Génie Electrique et Electronique, Soutenu le 14 Septembre 2014.

[15] Raphaël Soulard Philippe Carré : « Ondelettes quaternioniques pour la classification de textures », Laboratoire Xlim-SIC - CNRS UMR 6172, Université de Poitiers, pg 222-229, Janvier 2010, Bât. SP2MI, Téléport 2, Bvd. Marie et Pierre Curie BP 30179, 86962 Futuroscope Chasseneuil Cedex. e-mail : \{soulard,carre $\}$ sic.univ-poitiers.fr.

[16] Nicolas Le Bihan : «Traitement quaternionique des images couleur », Colloques sur le Traitement du Signal et des Images, 2003 - GRETSI (GRETSI, Groupe d'Etudes du Traitement du Signal et des Images).

[17] Raphaël Soulard Philippe Carré, « Ondelettes quaternioniques pour la classification de textures », Laboratoire Xlim-SIC - CNRS UMR 6172, Université de Poitiers, pg 222-229, Janvier 2010, Bât.

[18] Yann Gaudeau, Jean-Marie Moureaux, « Un schéma de compression avec pertes efficace pour les images médicales volumiques », 12ième journées d'étude et d'échange Compression et Représentation des Signaux Audiovisuels, coresa'2007, November 2007, montpellier, france. pp.cdrom.

[19] OUAFI Abdelkrim, « Compression d'images avec pertes par codages imbriqués, Proposition d'une optimisation de l'algorithme EZW », THESE Présentée pour l'obtention du Diplôme de Doctorat en Sciences en Electronique, université mohamed khider biskra faculte des sciences et technologie département d'électronique, 2008/2009.

[20] Tarun Kumar Rawat, Sumegha Yadav, « Transform Based Hybrid Image Compression Techniques in Conjunction with Fractal Image Compression Scheme »; International Journal of Advancements in Research \& Technology, Volume 1, Issue 4, April-2013 ISSN 2320-9119, IJOAR@ 2013 http://www.ijoar.org. 